preprint RUB-TPII-05/03

\title{
Sivers vs. Collins effect in azimuthal single spin asymmetries in pion production in SIDIS
}

\author{
A. V. Efremov ${ }^{a}$, K. Goeke ${ }^{b}$, P. Schweitzer ${ }^{c}$ \\ a Joint Institute for Nuclear Research, Dubna, 141980 Russia \\ ${ }^{b}$ Institut für Theoretische Physik II, Ruhr-Universität Bochum, Germany \\ ${ }^{c}$ Dipartimento di Fisica Nucleare e Teorica, Università degli Studi di Pavia, Pavia, Italy
}

June 2003

\begin{abstract}
Recently it has been argued that the transverse momentum dependent twist-2 Sivers distribution function does not vanish in QCD. Therefore both, the Collins and Sivers effects, should be considered in order to explain the azimuthal single spin asymmetries $A_{U L}$ in pion production in semi-inclusive deeply inelastic lepton scattering of a longitudinally polarized target. On the basis of presently available phenomenological information on the Sivers function we estimate that for those asymmetries $A_{U L}$ in the kinematic region of the HERMES experiments the Sivers effect can be neglected with respect to the Collins effect. It is argued that the same feature holds also for the COMPASS and CLAS experiments. This justifies theoretical approaches to understand the HERMES data on the basis of the Collins effect only.
\end{abstract}

Introduction. Recently, Brodsky, Hwang and Schmidt have shown that leading twist single spin asymmetries in semi-inclusive deeply inelastic scattering (SIDIS) can arise from a rescattering between the struck quark and the target remnant [1]. Collins has shown [2] that this rescattering mechanism is due to the Sivers effect [3], i.e. due to the existence of a (naively) T-odd transverse momentum dependent distribution function $f_{1 T}^{\perp}\left(x, \mathbf{p}_{\perp}^{2}\right)$, correcting his earlier proof that this distribution function vanishes [4] and legitimating phenomenological work $[5,6]$. The connection between such rescattering ("final state interactions") and a gauge-invariant definition of $f_{1 T}^{\perp}\left(x, \mathbf{p}_{\perp}^{2}\right)$ was further elaborated by Belitsky, Ji and Yuan [7].

In the light of $[1,2]$ it is not true anymore that the experimental HERMES results $[8,9,10,11]$ on azimuthal single spin asymmetries in SIDIS off a longitudinally (with respect to the beam) polarized target can be interpreted in terms of the Collins effect [4] only. Rather the Sivers effect should also be considered. The corresponding tree-level expressions were derived by Mulders et al. in [12, 13].

In Refs. $[14,15,16,17,18,19]$ it was aimed at a theoretical understanding of the HERMES data $[8,9,10]$ in terms of the Collins effect only, relying on the no more valid proof [4] that the Sivers distribution function vanishes. In principle these works should now be corrected to include the Sivers effect.

In this note we use presently available phenomenological information on the Sivers function by Anselmino et al. [5] (cf. [20]) to estimate the contribution of the Sivers effect to the azimuthal single spin asymmetries in the HERMES longitudinal target polarization experiments $[8,9,10]$ and find that it can be neglected compared to the Collins effect. We also argue that in the approach of the present authors $[18,19]$ the neglect of the Sivers effect was consistent and justified from a theoretical point of view.

Sivers effect in the HERMES longitudinal target polarization experiment. Let us consider the process $l p^{ \pm} \rightarrow l^{\prime} h X$ (see Fig. 1) where " $\pm "$ denotes the longitudinal (with respect to the beam) polarization of the proton target ("+" means polarization opposite to the beam direction). With $P$ denoting the momentum of the target proton, $l\left(l^{\prime}\right)$ denoting the momentum of the incoming (outgoing) lepton and $P_{h}$ the momentum of the produced hadron, the relevant kinematical variables are $s:=(P+l)^{2}, q=l-l^{\prime}$ with $Q^{2}=-q^{2}, x=\frac{Q^{2}}{2 P q}, y=\frac{P q}{P l}, z=\frac{P P_{h}}{P q}$. Let us consider the weighted cross section difference (cf. footnote 1 below)

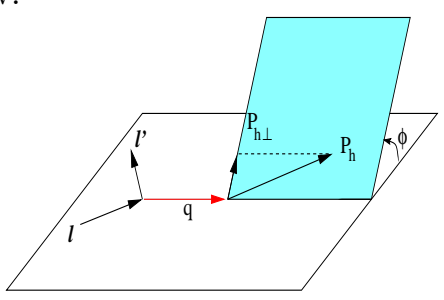

Figure 1: Kinematics of the process $l p \rightarrow l^{\prime} h X$ in the lab frame.

$$
\Delta \sigma^{\sin \phi k_{\perp} /\left\langle P_{h \perp}\right\rangle}(x)=\int \mathrm{d} z \mathrm{~d} y \mathrm{~d}^{2} \mathbf{P}_{h \perp} \sin \phi \frac{k_{\perp}}{\left\langle P_{h \perp}\right\rangle}\left(\frac{1}{S^{+}} \frac{\mathrm{d}^{5} \sigma^{+}}{\mathrm{d} x \mathrm{~d} y \mathrm{~d} z \mathrm{~d}^{2} \mathbf{P}_{h \perp}}-\frac{1}{S^{-}} \frac{\mathrm{d}^{5} \sigma^{-}}{\mathrm{d} x \mathrm{~d} y \mathrm{~d} z \mathrm{~d}^{2} \mathbf{P}_{h \perp}}\right),
$$


where $S^{ \pm}$denotes the modulus of the target polarization and $\sigma^{ \pm}$are the corresponding cross sections. The fact that in the HERMES experiment the cross sections were weighted without the transverse (with respect to the hard photon) momentum $k_{\perp}=\left|\mathbf{P}_{h \perp}\right| / z$ is not relevant for our discussion.

Assuming factorization the cross section asymmetry $\Delta \sigma^{\sin \phi k_{\perp} /\left\langle P_{h \perp}\right\rangle}$ was shown in a tree-level calculation up to $\mathcal{O}(1 / Q)$ to receive two contributions - one from the longitudinal and one from the transversal (with respect to the hard photon) component of the target spin $S[12,13]$

$$
\Delta \sigma^{\sin \phi k_{\perp} /\left\langle P_{h \perp}\right\rangle}(x)=\sigma_{U L}^{\sin \phi k_{\perp} /\left\langle P_{h \perp}\right\rangle}(x)+\sigma_{U T}^{\sin \phi k_{\perp} /\left\langle P_{h \perp}\right\rangle}(x) .
$$

The longitudinal part $\sigma_{U L}^{\sin \phi k_{\perp} /\left\langle P_{h \perp}\right\rangle}$ is due to the Collins effect only, while in the transversal part $\sigma_{U T}^{\sin \phi k_{\perp} /\left\langle P_{h \perp}\right\rangle}$ both Sivers and Collins effect contribute [13]

$$
\sigma_{U T}^{\sin \phi k_{\perp} /\left\langle P_{h \perp}\right\rangle}(x)=\sigma_{U T}^{\mathrm{Col}}(x)+\sigma_{U T}^{\mathrm{Siv}}(x)
$$

where

$$
\begin{aligned}
& \sigma_{U T}^{\mathrm{Col}}(x) \equiv-\frac{2}{S} \iint \mathrm{d} y \mathrm{~d} z\left\langle\frac{k_{\perp}}{\left\langle P_{h \perp}\right\rangle} \sin \left(\phi+\phi_{S}\right)\right\rangle_{O T O, \text { for } \phi_{S}=0}, \\
& \sigma_{U T}^{\mathrm{Siv}}(x) \equiv-\frac{2}{S} \iint \mathrm{d} y \mathrm{~d} z\left\langle\frac{k_{\perp}}{\left\langle P_{h \perp}\right\rangle} \sin \left(\phi-\phi_{S}\right)\right\rangle_{O T O, \text { for } \phi_{S}=0},
\end{aligned}
$$

in the notation of [13], or explicitly

$$
\begin{aligned}
& \sigma_{U T}^{\mathrm{Col}}(x)=-4 \pi \alpha^{2} s \int \mathrm{d} y \sin \Theta_{S} 2(1-y) Q^{-4} \sum_{a} e_{a}^{2} x h_{1}^{a}(x)\left\langle H_{1}^{\perp(1) a}\right\rangle, \\
& \sigma_{U T}^{\mathrm{Siv}}(x)=-4 \pi \alpha^{2} s \frac{M_{\mathrm{N}}}{\left\langle P_{h \perp}\right\rangle} \int \mathrm{d} y \sin \Theta_{S} 2\left(1-y+y^{2} / 2\right) Q^{-4} \sum_{a} e_{a}^{2} x f_{1 T}^{\perp(1) a}(x)\left\langle D_{1}^{a}\right\rangle,
\end{aligned}
$$

with $^{1}$ (recalling the relation $\mathbf{P}_{h \perp}=-z \mathbf{k}_{\perp}$ between the fragmenting quark and the produced hadron)

$$
\begin{aligned}
H_{1}^{\perp(1) a}(z) & =z^{2} \int \mathrm{d}^{2} \mathbf{k}_{\perp} \frac{\mathbf{k}_{\perp}^{2}}{2\left\langle P_{h \perp}\right\rangle^{2}} H_{1}^{\perp a}\left(z,-z \mathbf{k}_{\perp}\right), \\
f_{1 T}^{\perp(1) a}(x) & =\int \mathrm{d}^{2} \mathbf{p}_{\perp} \frac{\mathbf{p}_{\perp}^{2}}{2 M_{\mathrm{N}}^{2}} f_{1 T}^{\perp a}\left(x, \mathbf{p}_{\perp}\right) .
\end{aligned}
$$

In Eq. (5) $\sin \Theta_{S}=\left|\mathbf{S}_{T}\right| /|\mathbf{S}|=\left[\left(4 M_{\mathrm{N}}^{2} x^{2}\right)\left(1-y-M_{\mathrm{N}}^{2} x^{2} y^{2} / Q^{2}\right) /\left(Q^{2}+4 M_{\mathrm{N}}^{2} x^{2}\right)\right]^{1 / 2}$ characterizes the transverse (with respect to the photon) component of the target spin for longitudinal target polarization. $H_{1}^{\perp}$ is normalized according to the convention of Refs. $[12,13]$. In Eq. (4) $\phi_{S}$ denotes the azimuthal angle of the target spin around the photon direction with respect to lepton scattering plane. For a longitudinal polarization $\phi_{S}=-\pi$ (for "+" polarization in Eq. (1)) such that Sivers and Collins effect become indistinguishable. When integrating over $y$ and $z$ in Eqs. $(4,5)$ one has to consider the cuts $W^{2}>4 \mathrm{GeV}^{2}$ and $Q^{2}>1 \mathrm{GeV}^{2}$, $0.2<y<0.85$ and $0.2<z<0.7$ in the HERMES experiment.

Assuming a Gaussian distribution of transverse momenta ${ }^{2}$ for $H_{1}^{\perp}\left(z, \mathbf{P}_{h \perp}\right)$ one obtains for the cuts of the HERMES experiment

$$
\left\langle H_{1}^{\perp(1)}\right\rangle \equiv \int_{0.2}^{0.7} \mathrm{~d} z \int \mathrm{d}^{2} \mathbf{P}_{h \perp} \frac{k_{\perp}^{2}}{2\left\langle P_{h \perp}\right\rangle^{2}} H_{1}^{\perp}\left(z, \mathbf{P}_{h \perp}\right)=\frac{\left\langle k_{\perp}^{2}\right\rangle}{2\left\langle P_{h \perp}\right\rangle^{2}} \int_{0.2}^{0.7} \mathrm{~d} z H_{1}^{\perp}(z)=\frac{\left\langle k_{\perp}^{2}\right\rangle}{2\left\langle P_{h \perp}\right\rangle^{2}}\left\langle H_{1}^{\perp}\right\rangle .
$$

The $H_{1}^{\perp}(z)$ in $(7)$ is defined by the assumption of a Gaussian distribution of transverse momenta. It is this quantity which under certain assumptions was extracted by the present authors in Ref. [18] from the HERMES data [8, 9]. Assuming favoured fragmentation (i.e. in the following $\sum_{a}$ means the sum over the

\footnotetext{
${ }^{1}$ Note, that we use the notation of $[12,13]$ with the Collins function normalized with respect to $\left\langle P_{h \perp}\right\rangle$ instead of $m_{\pi}$, i.e. $\left[H_{1}^{\perp} /\left\langle P_{h \perp}\right\rangle\right]_{\text {here }}=\left[H_{1}^{\perp} / m_{\pi}\right]_{[12,13]}$. Correspondingly, it is $\left\langle P_{h \perp}\right\rangle$ which compensates the dimension of $k_{\perp}$ in the weight of the cross section asymmetry in Eqs. $(1,2,3)$ and in the definition (6). Note also the opposite definition of azimuthal angles in [13].

2 This assumption does not contradict the HERMES data, but it is not in agreement with the phenomenological considerations of Collins [4] or the model calculation of Bacchetta et al. in Ref. [21]. However, in a limited $z$-range $(0.2<z<0.7$ in the HERMES experiment) a relation of the kind (7) can always be assumed to hold with a sufficient accuracy for our purposes. Note that strictly speaking in the integration over transverse pion momenta also the experimental cuts should be considered $\left(\left|\mathbf{P}_{h \perp}\right|>50 \mathrm{MeV}\right.$ in the HERMES experiments).
} 
corresponding favoured flavours) we obtain for the ratio of "Sivers to Collins cross section asymmetry" the result

$$
\frac{\sigma_{U T}^{\mathrm{Siv}}(x)}{\sigma_{U T}^{\mathrm{Col}}(x)}=\frac{2 M_{\mathrm{N}}\left\langle P_{h \perp}\right\rangle}{\left\langle k_{\perp}^{2}\right\rangle} \frac{\left\langle D_{1}\right\rangle}{\left\langle H_{1}^{\perp}\right\rangle} \frac{\int \mathrm{d} y \sin \Theta_{S}\left(1-y+y^{2} / 2\right) / Q^{4}}{\int \mathrm{d} y \sin \Theta_{S}(1-y) / Q^{4}} \frac{\sum_{a} e_{a}^{2} x f_{1 T}^{\perp(1) a}(x)}{\sum_{b} e_{b}^{2} x h_{1}^{b}(x)} .
$$

Considering $\left\langle k_{\perp}^{2}\right\rangle=(4 / \pi)\left\langle k_{\perp}\right\rangle^{2}$ for a Gaussian distribution, using $\left\langle k_{\perp}\right\rangle=\left\langle P_{h \perp}\right\rangle /\langle z\rangle$ and inserting $\left\langle P_{h \perp}\right\rangle \approx$ $0.4 \mathrm{GeV}$ and $\langle z\rangle=0.41[8,9]$, and the value $\left\langle H_{1}^{\perp}\right\rangle /\left\langle D_{1}\right\rangle=(13.8 \pm 2.8) \%$ reported in [18] we obtain for the prefactors in Eq. (8) in the kinematics of the HERMES experiment

$$
\begin{array}{r}
\frac{2 M_{\mathrm{N}}\left\langle P_{h \perp}\right\rangle}{\left\langle k_{\perp}^{2}\right\rangle} \frac{\left\langle D_{1}\right\rangle}{\left\langle H_{1}^{\perp}\right\rangle} \\
1.03 \lesssim \frac{\int \mathrm{d} y \sin \Theta_{S}\left(1-y+y^{2} / 2\right) / Q^{4}}{\int \mathrm{d} y \sin \Theta_{S}(1-y) / Q^{4}} \lesssim 1.5 .
\end{array}
$$

As an estimate for the Sivers function we take the results extracted by Anselmino et al. in [5] (cf. [20]) from the E704 data [22] on single spin asymmetries in the process $p^{\uparrow} p \rightarrow \pi X$. If one assumes factorization, there are three possible non-perturbative mechanisms which could generate the observed effect: Collins effect, Sivers effect and a twist-3 mechanism [23]. Anselmino et al. tried to explain the E704 data under the assumption that the observed asymmetry is due to the Sivers effect only. Under this assumption the following fit of the Sivers function to the data was reported [5] (cf. [20])

$$
\begin{aligned}
& f_{1 T}^{\perp(1) u}(x)=0.81 x^{2.70}(1-x)^{4.54} \\
& f_{1 T}^{\perp(1) d}(x)=-0.27 x^{2.12}(1-x)^{5.10} \\
& f_{1 T}^{\perp(1) \bar{q}}(x)=0 .
\end{aligned}
$$

The result (10) refers to a scale which is of the order of magnitude of the large transverse momentum of the produced pions - typically $(2-3) \mathrm{Gev}$, i.e. comparable to the $Q^{2}$-region explored in the

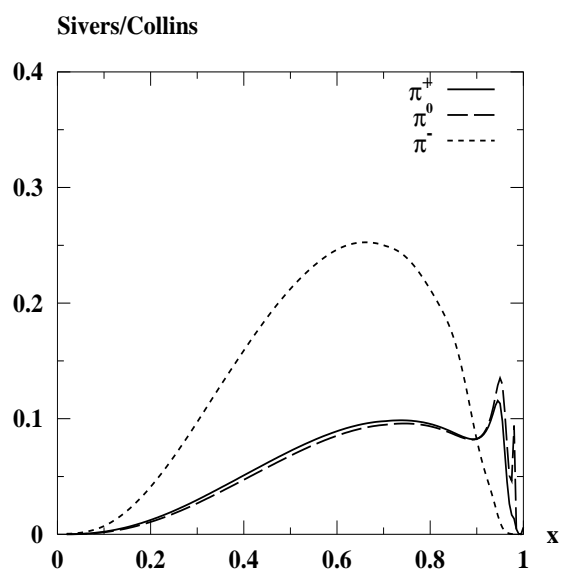

Figure 2: The "ratio of Sivers to Collins effect" as defined in Eq. (8) vs. $x$ for the kinematics of the HERMES experiment HERMES experiment. Using the estimate (10) for the Sivers function we obtain for the ratio of "Sivers to Collins effect" as defined in Eq. (8) the result shown in Fig. 2. (For the transversity distribution $h_{1}^{a}(x)$ we take the prediction of the Chiral Quark Soliton Model [24] LO-evolved to $Q^{2}=4 \mathrm{GeV}^{2}$ as used in [18, 19].)

As clarified in [2, 7] (see also [25]) the Wilson-link required to ensure gauge invariance of the expression for the Sivers-function is process-dependent and implies opposite signs for $f_{1 T}^{\perp}$ in SIDIS and in the Drell-Yan process. The connection between $f_{1 T}^{\perp}$ in SIDIS and in $p^{\uparrow} p \rightarrow \pi X$ has not been clarified yet. ${ }^{3}$ In Fig. 2 it is assumed that $f_{1 T}^{\perp}$ has the same sign in these processes (otherwise the result is to be understood as the modulus of the ratio of "Sivers to Collins effect").

The result in Fig. 2 means that relying on the estimate (10) the contribution of the Sivers effect to the azimuthal asymmetries from a longitudinally polarized target can safely be neglected for the kinematics of the HERMES experiment. We observe that the Sivers effect contributes for $0.023<x<0.4$ (the HERMES $x$-cuts) about $(2-3) \%$ to the transversal part of the asymmetry $A_{U L}^{\sin \phi}$ in the case of $\pi^{+}$and $\pi^{0}$. The transversal part provides a negative and in absolute values smaller contribution the total asymmetry $A_{U L}^{\sin \phi}$ compared to the longitudinal part [18]. (According to our estimates the Sivers effect would contribute about $10 \%$ to the transversal part in the case of $\pi^{-}$where, however, unfavoured fragmentation effects play a much more important role [16]. In the HERMES experiment $\pi^{-}$azimuthal asymmetries were found consistent with zero.) It should be noted that the suppression of the Sivers effect with respect to the Collins effect is not a kinematical effect fore the respective prefactors are not small, see Eq. (9).

One could be tempted to interpret (10) as an upper bound for the Sivers function fore it quantifies the right portion of the Sivers effect needed to explain the E704 data in terms of this effect only. However, as already mentioned, there are two more effects namely the Collins effect and the twist-3 mechanism proposed in [23] which could give rise to the effect observed in the E704 experiment. One could imagine a situation where

\footnotetext{
${ }^{3}$ For a discussion of the processes $p^{\uparrow} p \rightarrow \pi X$ and $e \vec{p} \rightarrow \pi X$ at large transverse momenta, which can be described by related (twist-3) functions [25], see Ref. [26].
} 
the three effects were sizeable, but contributed to the net result with different signs and partially canceled each other. Therefore, we cannot consider the result of Fig. 2 as a strict upper bound for the contribution of the Sivers effect to the HERMES azimuthal asymmetries from a longitudinally polarized target. Rather we can interpret the result of Fig. 2 as an indication that the Sivers effect is of little importance in the corresponding HERMES experiments [8, 9, 10].

In this context it is interesting to remark that Anselmino et al. also made the attempt to understand the E704 data in terms of the Collins effect only, observing that in principle this is possible [27]. By comparing the $H_{1}^{\perp}$ of the present authors [18] (which explains the HERMES data [8,9] by the Collins effect only) to the $H_{1}^{\perp}$ of Anselmino et al. [27] (which explains the E704 data [22] by the Collins effect only), we come to the following conclusion: The $H_{1}^{\perp}$ of the present authors [18] could account for roughly half the effect observed in the E704 experiment [22]. In particular, the Collins effect contributes to the E704 data with a positive $\operatorname{sign}^{4}$. This would exclude the possibility of a partial cancellation of Sivers and Collins effect. Still there is the twist-3 mechanism [23] which does not allow us to consider the result in Fig. 2 as a definite bound for the contribution of the Sivers effect.

Finally we remark that the attempt to explain HERMES data $[8,9,10]$ in terms of the Sivers effect only, would require a Sivers function one order of magnitude larger and of opposite sign than in the E704 experiment (however, cf. the discussion above). So these two experiments are only compatible with each other if the Collins effect plays an important role.

Calculations in the quark-diquark models with gluon exchange [29, 30] suggest a somehow larger Siversfunction than the estimate in Eq. (10). However, it should be noted that even a Sivers-function significantly larger (up to an order of magnitude) than (10) still would yield a negligible effect, at least for positive and neutral pions. Thus, qualitatively our conclusions are not altered in the light of the results reported in $[29,30]$.

CLAS and COMPASS experiments. Azimuthal asymmetries will also be studied in the CLAS and COMPASS experiments. We find that the suppression effect of the Sivers with respect to the Collins effect in asymmetries from a longitudinally target is only weakly sensitive to cuts. The suppression is stronger with increasing scale because $f_{1 T}^{\perp a}$ decreases with increasing scale more rapidly than $h_{1}^{a}$ [31]. This means that dedicated experiments with longitudinally polarized targets at CLAS and COMPASS can also be interpreted solely on the basis of the Collins mechanism. (Predictions for CLAS are presented by the present authors in [32] and those for COMPASS will be given elsewhere).

Sivers effect in SIDIS with transversely polarized target. In the case of a longitudinally polarized target both Sivers and Collins effect contribute. In contrast, a transversely polarized target allows a clean separation of these effects by using appropriate weights [13]

$$
\begin{aligned}
A_{U T}^{\sin \left(\phi \mp \phi_{s}\right) k_{\perp}}(x) & =\frac{\int \mathrm{d} z \mathrm{~d} y \mathrm{~d}^{2} \mathbf{P}_{h \perp} \sin \left(\phi \mp \phi_{s}\right) k_{\perp}\left(\frac{1}{S^{\uparrow}} \frac{\mathrm{d}^{5} \sigma^{\uparrow}}{\mathrm{d} x \mathrm{~d} y \mathrm{~d} z \mathrm{~d}^{2} \mathbf{P}_{h \perp}}-\frac{1}{S^{\downarrow}} \frac{\mathrm{d}^{5} \sigma^{\downarrow}}{\mathrm{d} x \mathrm{~d} y \mathrm{~d} z \mathrm{~d}^{2} \mathbf{P}_{h \perp}}\right)}{\frac{1}{2} \int \mathrm{d} z \mathrm{~d} y \mathrm{~d}^{2} \mathbf{P}_{h \perp}\left(\frac{\mathrm{d}^{5} \sigma^{\uparrow}}{\mathrm{d} x \mathrm{~d} y \mathrm{~d} z \mathrm{~d}^{2} \mathbf{P}_{h \perp}}+\frac{\mathrm{d}^{5} \sigma^{\downarrow}}{\mathrm{d} x \mathrm{~d} y \mathrm{~d} z \mathrm{~d}^{2} \mathbf{P}_{h \perp}}\right)} \\
& \propto \begin{cases}f_{1 T}^{\perp} D_{1} & \text { for "-" (Sivers effect) } \\
h_{1} H_{1}^{\perp} & \text { for "+"(Collins effect) })\end{cases}
\end{aligned}
$$

where $k_{\perp}=\left|\mathbf{P}_{h \perp}\right| / z$. In the case of transverse polarization the azimuthal angle of the spin vector differs from event to event and has to be determined from the data. More specifically the result for the asymmetry reads $[13]$

$$
A_{U T}^{\sin \left(\phi-\phi_{s}\right) k_{\perp} / M_{\mathrm{N}}}(x)=\frac{2 \int \mathrm{d} y \cos \Theta_{S}\left(1-y+y^{2} / 2\right) Q^{-4} \sum_{a} e_{a}^{2} x f_{1 T}^{\perp(1) a}(x)\left\langle D_{1}^{a}\right\rangle}{\int \mathrm{d} y\left(1-y+y^{2} / 2\right) Q^{-4} \sum_{b} e_{b}^{2} x f_{1}^{b}(x)\left\langle D_{1}^{b}\right\rangle}
$$

Let us estimate the azimuthal asymmetry $A_{U T}^{\sin \left(\phi-\phi_{s}\right) k_{\perp} / M_{\mathrm{N}}}$ on the basis of the results (10) from Ref. [5]. ${ }^{5}$ Assuming favoured fragmentation we obtain the result shown in Fig. 3a.

\footnotetext{
${ }^{4}$ Hereby we assume universality of $H_{1}^{\perp}$ in SIDIS and $p^{\uparrow} p \rightarrow \pi X$. In Ref. [25] it has recently been argued that there might be no simple relation between the Collins-function in SIDIS and $e^{+} e^{-}$annihilation. (However, see also [28].) The relation between $H_{1}^{\perp}$ in SIDIS and $p^{\uparrow} p \rightarrow \pi X$ has not been clarified yet.

${ }^{5}$ Such an estimate has already been given in [20] in Fig. 5 and Fig. 6, which show respectively the numerator and denominator of Eq. (12) (without $z$-average) as functions of $x$ and $z$ in 3-D plots.
} 

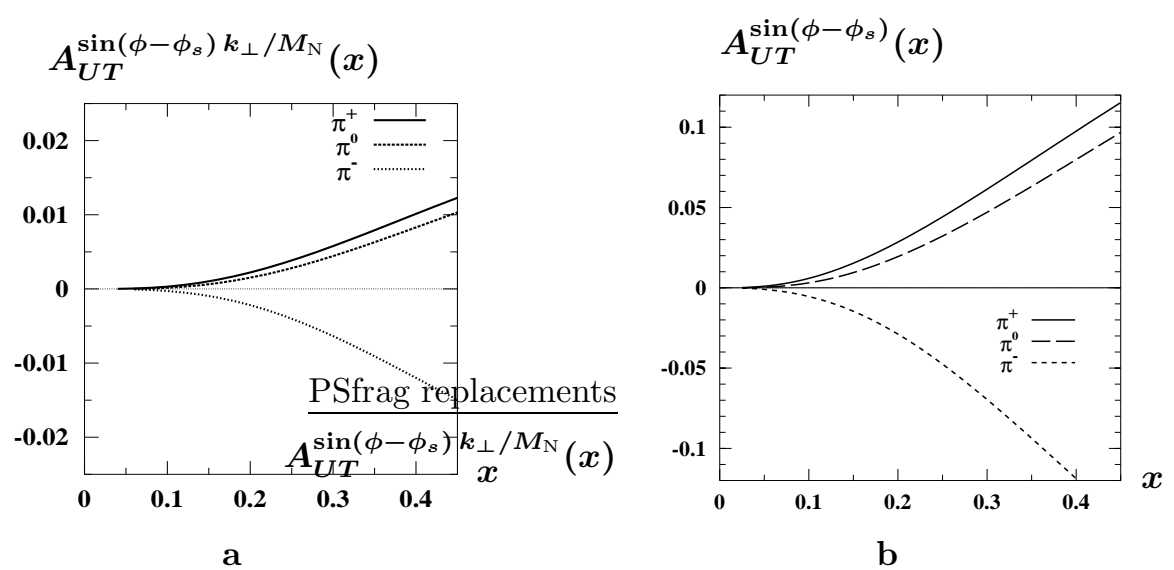

Figure 3: (a) The single spin azimuthal asymmetry $A_{U T}^{\sin \left(\phi-\phi_{s}\right) k_{\perp} / M_{\mathrm{N}}}(x)$ for $\pi^{ \pm}$and $\pi^{0}$ for the HERMES kinematics as a function of $x$. Note that the weight $k_{\perp} / M_{\mathrm{N}}$ provides an artificial suppression, see text.

(b) The single spin azimuthal asymmetry $A_{U T}^{\sin \left(\phi-\phi_{s}\right)}(x)$ (i.e. weighted without the factor $k_{\perp} / M_{\mathrm{N}}$ ) for the HERMES kinematics as a function of $x$.

The $k_{\perp}$-weighted asymmetry $A_{U T}^{\sin \left(\phi-\phi_{s}\right) k_{\perp} / M_{\mathrm{N}}}$ is about $1 \%$. However, this does not mean that the effect itself is small because the $k_{\perp} / M_{\mathrm{N}}$-factor in the weight introduces an artificial suppression of the effect. In $[5,20]$ the result $\left\langle k_{T}^{2}(x)\right\rangle^{1 / 2}=0.47 x^{0.68}(1-x)^{0.48} M_{\mathrm{N}}$ was used from a parton model based analysis [33]. This result implies that $\left\langle k_{T}^{2}(x)\right\rangle^{1 / 2} \lesssim 0.2 M_{\mathrm{N}}$ for all $x$. In order to compare the effect "more directly" to the asymmetries $A_{U L}^{\sin \phi} \sim(3-4) \%$ measured by HERMES $[8,9,10]$ we consider the asymmetry weighted without the factor $k_{\perp} / M_{\mathrm{N}}$, which we estimate as follows (cf. Appendix)

$$
A_{U T}^{\sin \left(\phi-\phi_{s}\right)}(x) \approx \frac{2 M_{\mathrm{N}}}{\left\langle k_{T}\right\rangle} A_{U T}^{\sin \left(\phi-\phi_{s}\right) k_{T} / M_{\mathrm{N}}}(x) .
$$

We roughly approximate $\left\langle k_{T}\right\rangle \approx\left\langle k_{T}^{2}\right\rangle^{1 / 2}$ and take $\left\langle k_{T}^{2}\right\rangle=\left\langle k_{T}^{2}(x)\right\rangle$ from [33]. (It is consistent and necessary to use $f_{1 T}^{\perp}$ from [5, 20] in connection with $\left\langle k_{T}^{2}\right\rangle$ from [33] because the latter was used explicitly in Ref. [5] to fit $f_{1 T}^{\perp}$ under the above-mentioned assumptions.)

The result for the asymmetry weighted without the factor $k_{\perp} / M_{\mathrm{N}}, A_{U T}^{\sin \left(\phi-\phi_{s}\right)}(x)$, is shown in Fig. 3b. We see that the effect itself is not small. However, the corresponding (non- $k_{\perp}$-weighted) $A_{U T}^{\sin \left(\phi+\phi_{s}\right)}(x) \propto h_{1}^{a} H_{1}^{\perp a}$ Collins effect asymmetry is larger, namely of $\mathcal{O}(20 \%)$ [34].

Calculations based on a quark-diquark model approach yield an $A_{U T}^{\sin \left(\phi-\phi_{s}\right)}(x)$ of comparable magnitude at large (in the HERMES kinematics) $x \sim 0.3$, but substantially more sizeable than the result in Fig. 3b in the region $x \lesssim 0.2$ [30]. We emphasize that the results in Figs. 3a and 3b can only be viewed as rough estimates with the following significance: If HERMES measured $A_{U T}^{\sin \left(\phi-\phi_{s}\right) k_{\perp} / M_{\mathrm{N}}}$ or $A_{U T}^{\sin \left(\phi-\phi_{s}\right)}$ of comparable order of magnitude as the results in Figs. $3 \mathrm{a}$ and $3 \mathrm{~b}$, then the arguments and estimates given in the context of Fig. 2 would experimentally be justified.

Comment on the calculations of $A_{U L}$ by the present authors. In Refs. [18, 19] it was aimed at describing the HERMES data $[8,9,10]$ in terms of the Collins effect only.

The approach of $[18,19]$ consists in combining results from the instanton model of the QCD vacuum [35] and from the chiral quark-soliton model $[36,37]$ for the nucleon chirally odd distribution functions $h_{1}^{a}(x)$ and $h_{L}^{a}(x)[24,38]$. This approach is consistent because the chiral quark-soliton model was derived from the instanton vacuum model. A small parameter which played an important role in this derivation is the instanton packing fraction - the ratio of the average instanton size $\rho$ to the average separation $R$ of instantons (in Euclidean space-time). Numerically $\rho / R \sim 1 / 3$ with $\rho \approx(600 \mathrm{MeV})^{-1}$.

The neglect of Sivers function $f_{1 T}^{\perp a}$ in the approach of Refs. [18, 19] is fully consistent for the following reason. In Ref. [39] Pobylitsa showed that in a large class of chiral models " $T$-odd" distribution functions are strictly zero. In particular in the chiral quark-soliton model $f_{1 T}^{\perp a}=0$. This can be understood by considering that in QCD $f_{1 T}^{\perp a}$ does not vanish under time reversal only due to the non-trivial transversal part of the Wilson line entering the definition of $f_{1 T}^{\perp}\left(x, \mathbf{p}_{\perp}^{2}\right)[2,7]$. Most chiral models are based on quark and Goldstone-boson degrees of freedom, and the modeling of the Wilson-line is beyond the scope of such models. 
(By itself this does not mean that the Sivers function is necessarily small or even zero in nature. Indeed, other models which explicitly take into account gluonic degrees of freedom have no problem with modeling the Wilson-line. One example are the quark-diquark models with gluon exchange of Refs. $[1,29,30]$.)

However, if one considers that in the chiral quark-soliton model $f_{1 T}^{\perp a}=0$ and that this model in a certain sense corresponds to the zeroth order in the parameter $\rho / R$ of the instanton model [40], then one arrives at the conclusion that $f_{1 T}^{\perp a}$ is suppressed in the parameter $\rho / R$. This conclusion is drawn here indirectly and should, of course, be reinvestigated in the instanton vacuum model using the methods developed in [41].

For the calculations of $A_{U L}$ at HERMES by the present authors $[18,19]$ the information that $f_{1 T}^{\perp a}$ is strictly zero in the chiral quark-soliton model (and suppressed in the instanton vacuum model) is, from the theoretical point of view, fully sufficient to neglect Sivers effect. As we have seen above, this is supported also by phenomenology.

Conclusions. It was shown that on the basis of presently available phenomenological information on the Sivers function [5] the contribution of the Sivers effect to single spin asymmetries from a longitudinally polarized target can be neglected with respect to the Collins effect. This result means that the HERMES data $[8,9,10]$ indeed provide us with first insights into the chirally odd structure of the nucleon, and that the theoretical efforts to understand these data in terms of the Collins effect only $[14,15,16,17,18,19]$ were justified. The same applies to CLAS and COMPASS kinematics, which is an encouraging result for these experiments where (most of the beam-time) longitudinally polarized targets will be used.

Single spin azimuthal asymmetries from transversely polarized targets allow an unambiguous distinction of the Collins and Sivers effect. Such asymmetries are presently studied by the HERMES [11] and COMPASS collaborations. Relying on the information on the Sivers function from Ref. [5] one can estimate the Sivers effect on single spin asymmetries from a transversely polarized proton target to be about $5 \%$. This must not be considered as an absolute prediction, rather as a rough indication for the size of the effect, and will serve as a thorough experimental test of the considerations presented in this note. Our predictions for the Collins effect in single spin asymmetries from a transversely polarized target will be presented elsewhere.

Acknowledgement. We are grateful to P. V. Pobylitsa and H.Avakian for fruitful discussions. A. E. is partially supported by INTAS grant 00/587 and RFBR grant 03-02-16816. This work has partly been performed under the contract HPRN-CT-2000-00130 of the European Commission. The work is partially supported by BMBF and DFG of Germany and by the COSY-Juelich project.

Appendix. Explicit formulae for azimuthal asymmetries weighted with an appropriate power of transverse momentum were derived in [13]. In asymmetries weighted without an appropriate power of $k_{\perp}$ the transverse momenta in the unintegrated distribution and fragmetation functions, in our case $f_{1 T}^{\perp a}\left(x, \mathbf{k}_{T}^{2}\right)$ and $D_{1}\left(z, \mathbf{K}_{T, D}^{2}\right)$, remain convoluted. (For a discussion of the meaning of unintegrated distribution functions in QCD, see Ref. [42].) In order to deal with this case two approaches have been followed in the literature.

One approach consists in approximating (in the lucid notation of [13])

$$
\left\langle\sin \left(\phi-\phi_{S}\right)\right\rangle_{\text {OTO }} \approx \frac{2 M_{\mathrm{N}}}{\left\langle k_{T}\right\rangle}\left\langle\frac{k_{\perp}}{M_{\mathrm{N}}} \sin \left(\phi-\phi_{S}\right)\right\rangle_{\text {OTO }} .
$$

This approach was chosen in Refs. $[14,16]$ in studies of $A_{U L}$ asymmetries, and in Ref. [30] in studies of the $A_{U T}$ asymmetry which we consider here. Eq. (13) is just the estimate (14) in a different notation.

Another approach consists in directly evaluating the asymmetries with a Gaussian ansatz (cf. footnote 2)

$$
F\left(x, \mathbf{k}_{T}^{2}\right)=F(x) \frac{\exp \left(-\mathbf{k}_{T}^{2} /\left\langle\mathbf{k}_{T}^{2}\right\rangle\right)}{\pi\left\langle\mathbf{k}_{T}^{2}\right\rangle}
$$

where $F\left(x, \mathbf{k}_{T}^{2}\right)$ denotes a generic unintegrated distribution or fragmentation function. The normalization factors in Eq. (15) are such that $\int \mathrm{d}^{2} \mathbf{k}_{T} F\left(x, \mathbf{k}_{T}^{2}\right)=F(x)$ holds. Under the assumption (15) we obtain

$$
\begin{aligned}
A_{U T}^{\sin \left(\phi-\phi_{s}\right)}(x) \stackrel{\text { Gauss }}{=} & B_{\text {corr }} \times\{\text { the result in Eq. (13) }\}, \\
& B_{\text {corr }}=\frac{\pi}{4} \cdot \frac{1}{2} \cdot\left(1+\frac{\left\langle K_{T, D}^{2}\right\rangle}{\left\langle k_{T}^{2}\right\rangle}\right)^{-1 / 2} .
\end{aligned}
$$


The transverse momenta of the fragmenting quarks are related to those of the produced hadrons by $\left\langle K_{T, D}^{2}\right\rangle \approx$ $\left\langle P_{h \perp}^{2}\right\rangle /\left\langle z^{2}\right\rangle$. Eq. (16) can be derived following Ref. [12], where explicit examples of similar calculations based on the ansatz (15) can be found. This approach was chosen in the case of $A_{U L}$ asymmetries in $[17,18,19]$.

The appearance of a "correction factor" between the heuristic estimate in Eq. (14) and the consistent calculation under a Gaussian assumption is not surprizing. In the present case one finds an $x$-dependent $B_{\text {corr }} \lesssim 0.1$ (for $\left\langle k_{T}^{2}\right\rangle$ from [33] and $\left\langle K_{T, D}^{2}\right\rangle \approx\left\langle P_{h \perp}^{2}\right\rangle /\left\langle z^{2}\right\rangle$ from the HERMES experiment). I.e. the second method would yield a substantially smaller result. Both approaches are, of course, heuristic and it is not clear which could be more realistic and reliable. In this work we preferred the approach based on Eq. (14) in order to directly compare to other calculations reported in literature [30].

Note that $B_{T} \neq 1$ means that the estimate (14) is not compatible with a Gaussian distribution of transverse momenta. Therefore we approximate $\left\langle k_{T}\right\rangle^{2} \approx\left\langle k_{T}^{2}\right\rangle$ in the sequence of Eq. (13), as there would be no justification to use, e.g., the relation $\left\langle k_{T}\right\rangle^{2}=\pi\left\langle k_{T}^{2}\right\rangle / 4$ valid for a Gaussian distribution only.

It should be stressed that azimuthal asymmetries offer - beyond insights into the T-odd Collins and Sivers mechanisms - also the opportunity to learn about transverse quark momenta in hadrons. An analysis of data from HERMES (and other experiments) using both, weights with and without an explicit factor $k_{T}=\left|\mathbf{P}_{h \perp}\right| / z$, could provide valuable phenomenological insights. From a strict theoretical point of view, however, the weighting with appropriate factors of $k_{T}$ is preferable [13].

\section{References}

[1] S. J. Brodsky, D. S. Hwang and I. Schmidt, Phys. Lett. B 530 (2002) 99 [arXiv:hep-ph/0201296].

[2] J. C. Collins, Phys. Lett. B 536 (2002) 43 [arXiv:hep-ph/0204004].

[3] D. W. Sivers, Phys. Rev. D 41 (1990) 83 [Annals Phys. 198 (1990) 371]; Phys. Rev. D 43 (1991) 261.

[4] J. C. Collins, Nucl. Phys. B 396 (1993) 161 [arXiv:hep-ph/9208213].

[5] M. Anselmino, M. Boglione and F. Murgia, Phys. Lett. B 362 (1995) 164 [arXiv:hep-ph/9503290]. M. Anselmino and F. Murgia, Phys. Lett. B 442 (1998) 470 [arXiv:hep-ph/9808426].

[6] D. Boer, Phys. Rev. D 60 (1999) 014012 [arXiv:hep-ph/9902255].

[7] A. V. Belitsky, X. Ji and F. Yuan, Nucl. Phys. B 656 (2003) 165 [arXiv:hep-ph/0208038]. X. D. Ji and F. Yuan, Phys. Lett. B 543 (2002) 66 [arXiv:hep-ph/0206057].

[8] A. Airapetian et al. [HERMES Collaboration], Phys. Rev. Lett. 84 (2000) 4047 [arXiv:hep-ex/9910062]. H. Avakian [HERMES Collaboration], Nucl. Phys. Proc. Suppl. 79 (1999) 523.

[9] A. Airapetian et al. [HERMES Collaboration], Phys. Rev. D 64 (2001) 097101 [arXiv:hep-ex/0104005].

[10] A. Airapetian et al. [HERMES Collaboration], Phys. Lett. B 562 (2003) 182 [arXiv:hep-ex/0212039].

[11] N. C. Makins and M. Düren [HERMES Collaboration], Acta Phys. Polon. B 33 (2002) 3737. N. C. Makins [HERMES Collaboration], Nucl. Phys. A 711 (2002) 41 [arXiv:hep-ex/0209035].

[12] P. J. Mulders and R. D. Tangerman, Nucl. Phys. B461 (1996) 197 [Erratum-ibid. B484 (1996) 538] [arXiv:hep-ph/9510301].

[13] D. Boer and P. J. Mulders, Phys. Rev. D 57 (1998) 5780 [arXiv:hep-ph/9711485].

[14] E. De Sanctis, W. D. Nowak and K. A. Oganesian, Phys. Lett. B 483 (2000) 69 [arXiv:hep-ph/0002091]; V. A. Korotkov, W. D. Nowak and K. A. Oganesian, Eur. Phys. J. C 18 (2001) 639 [arXiv:hepph/0002268]; K. A. Oganessian, N. Bianchi, E. De Sanctis and W. D. Nowak, Nucl. Phys. A 689 (2001) 784 [arXiv:hep-ph/0010261].

[15] M. Anselmino and F. Murgia, Phys. Lett. B 483 (2000) 74 [arXiv:hep-ph/0002120].

[16] B. Q. Ma, I. Schmidt and J. J. Yang, Phys. Rev. D 66 (2002) 094001 [arXiv:hep-ph/0209114]; Phys. Rev. D 65 (2002) 034010 [arXiv:hep-ph/0110324]; Phys. Rev. D 63 (2001) 037501 [arXiv:hep-ph/0009297].

[17] A. V. Efremov, K. Goeke, M. V. Polyakov and D. Urbano, Phys. Lett. B 478 (2000) 94, hep-ph/0001119. 
[18] A. V. Efremov, K. Goeke and P. Schweitzer, Phys. Lett. B 522 (2001) 37 [Erratum-ibid. B 544 (2002) 389] [arXiv:hep-ph/0108213].

[19] A. V. Efremov, K. Goeke and P. Schweitzer, Eur. Phys. J. C 24 (2002) 407 [arXiv:hep-ph/0112166]; Nucl. Phys. A 711 (2002) 84; Acta Phys. Polon. B 33 (2002) 3755 [arXiv:hep-ph/0206267].

[20] M. Boglione and P. J. Mulders, Phys. Rev. D 60 (1999) 054007, hep-ph/9903354.

[21] A. Bacchetta, R. Kundu, A. Metz and P. J. Mulders, Phys. Rev. D 65 (2002) 094021 [arXiv:hep$\mathrm{ph} / 0201091]$.

[22] D. L. Adams et al. [FNAL-E704 Collaboration], Phys. Lett. B 264 (1991) 462.

[23] A. V. Efremov and O. V. Teryaev, Yad. Fiz. 39 (1984) 1517; Phys. Lett. B 150 (1985) 383.

J. W. Qiu and G. Sterman, Phys. Rev. Lett. 67 (1991) 2264; Nucl. Phys. B 378 (1992) 52.

A. Efremov, V. Korotkiian and O. Teryaev, Phys. Lett. B 348 (1995) 577.

V. M. Korotkiian and O. V. Teryaev, Phys. Rev. D 52 (1995) 4775.

J. W. Qiu and G. Sterman, Phys. Rev. D 59 (1999) 014004.

Y. Kanazawa and Y. Koike, Phys. Lett. B 478 (2000) 121; Phys. Lett. B 490 (2000) 99.

[24] P. V. Pobylitsa and M. V. Polyakov, Phys. Lett. B 389 (1996) 350 [arXiv:hep-ph/9608434].

P. Schweitzer, D. Urbano, M. V. Polyakov, C. Weiss, P. V. Pobylitsa and K. Goeke, Phys. Rev. D 64 (2001) 034013 [arXiv:hep-ph/0101300].

[25] D. Boer, P. J. Mulders and F. Pijlman, arXiv:hep-ph/0303034.

[26] Y. Koike, Talk given at 15th International Spin Physics Symposium (SPIN 2002), Long Island, New York, 9-14 Sep 2002, arXiv:hep-ph/0210396.

[27] M. Anselmino, M. Boglione and F. Murgia, Phys. Rev. D 60 (1999) 054027 [arXiv:hep-ph/9901442].

[28] A. Metz, Phys. Lett. B 549 (2002) 139.

[29] S. J. Brodsky, D. S. Hwang and I. Schmidt, Phys. Lett. B 553 (2003) 223; Nucl. Phys. B 642 (2002) 344. D. Boer, S. J. Brodsky and D. S. Hwang, Phys. Rev. D 67 (2003) 054003.

[30] L. P. Gamberg, G. R. Goldstein and K. A. Oganessyan, Phys. Rev. D 67 (2003) 071504 [arXiv:hep$\mathrm{ph} / 0301018]$.

[31] A. A. Henneman, D. Boer and P. J. Mulders, Nucl. Phys. B 620 (2002) 331 [arXiv:hep-ph/0104271].

[32] A. V. Efremov, K. Goeke and P. Schweitzer, Phys. Rev. D 67 (2003) 114014 [arXiv:hep-ph/0208124].

[33] J. D. Jackson, G. G. Ross and R. G. Roberts, Phys. Lett. B 226 (1989) 159.

[34] A. V. Efremov, K. Goeke and P. Schweitzer, in preparation.

[35] D. Diakonov and V. Y. Petrov, Nucl. Phys. B 245 (1984) 259.

[36] D. Diakonov, V. Y. Petrov and P. V. Pobylitsa, Nucl. Phys. B 306 (1988) 809.

[37] For a review see: C. V. Christov et al., Prog. Part. Nucl. Phys. 37 (1996) 91 [arXiv:hep-ph/9604441].

[38] B. Dressler and M. V. Polyakov, Phys. Rev. D 61 (2000) 097501 [arXiv:hep-ph/9912376].

[39] P. V. Pobylitsa, arXiv:hep-ph/0212027.

[40] D. Diakonov, V. Petrov, P. V. Pobylitsa, M. V. Polyakov and C. Weiss, Nucl. Phys. B 480 (1996) 341 [arXiv:hep-ph/9606314].

[41] D. Diakonov, M. V. Polyakov and C. Weiss, Nucl. Phys. B 461 (1996) 539 [arXiv:hep-ph/9510232].

[42] J. C. Collins, Acta Phys. Polon. B 34 (2003) 3103 [arXiv:hep-ph/0304122]. 